\title{
An Approach to Study the Interactions between Ellagitannins and Oxygen during Oak Wood Aging
}

Ignacio García-Estévez, Cristina Alcalde-Eon, Ana María Martínez-Gil, Julian C. RivasGonzalo, M. Teresa Escribano-Bailon, Ignacio Nevares, and Maria del Alamo-Sanza

J. Agric. Food Chem., Just Accepted Manuscript • Publication Date (Web): 23 May 2017

Downloaded from http://pubs.acs.org on May 28, 2017

\section{Just Accepted}

"Just Accepted" manuscripts have been peer-reviewed and accepted for publication. They are posted online prior to technical editing, formatting for publication and author proofing. The American Chemical Society provides "Just Accepted" as a free service to the research community to expedite the dissemination of scientific material as soon as possible after acceptance. "Just Accepted" manuscripts appear in full in PDF format accompanied by an HTML abstract. "Just Accepted" manuscripts have been fully peer reviewed, but should not be considered the official version of record. They are accessible to all readers and citable by the Digital Object Identifier (DOI®). "Just Accepted" is an optional service offered to authors. Therefore, the "Just Accepted" Web site may not include all articles that will be published in the journal. After a manuscript is technically edited and formatted, it will be removed from the "Just Accepted" Web site and published as an ASAP article. Note that technical editing may introduce minor changes to the manuscript text and/or graphics which could affect content, and all legal disclaimers and ethical guidelines that apply to the journal pertain. ACS cannot be held responsible for errors or consequences arising from the use of information contained in these "Just Accepted" manuscripts. 


\section{An Approach to Study the Interactions between Ellagitannins and Oxygen during}

\section{Oak Wood Aging}

Ignacio García-Estévez ${ }^{1 *}$, Cristina Alcalde-Eon ${ }^{1 \ddagger}$, Ana María Martínez-Gil ${ }^{2}$, Julián C. RivasGonzalo $^{1}$, M. Teresa Escribano-Bailón ${ }^{1}$, Ignacio Nevares ${ }^{2}$, María del Alamo-Sanza ${ }^{2}$ *

${ }^{1}$ Grupo de Investigación en Polifenoles (GIP), Facultad de Farmacia, University of Salamanca, Campus Miguel de Unamuno, E 37007 Salamanca, Spain

${ }^{2}$ Grupo de Investigación UVaMOX, ETS Ingenierías Agrarias, Universidad de Valladolid,

Campus La Yutera, E 34001, Palencia, Spain. Fax +34-979-108302; phone +34-979-108355

$$
\text { e-mail*delalamo@ga.uva.es }
$$

\footnotetext{
${ }^{\ddagger}$ Both authors equally contributed to the manuscript
} 


\section{ABSTRACT}

2 During the aging of red wine in oak wood barrels, or in alternative aging systems, 3 interactions between the compounds released from wood, the compounds of the wine 4 and oxygen can take place. The main objective of the present work was to study 5 oxygen-ellagitannin interactions by monitoring their levels in three model systems all

6 containing the same amounts of French oak chips and only differing in the oxygen

7 content: total absence, only the oxygen released from the chips and air-saturated (model

8 systems $\mathbf{F}$, OW and $\mathbf{O S}$, respectively). This study has highlighted the influence of 9 oxygen in the ellagitannins evolution and the relevance of the oxygen trapped into the 10 oak chips, reporting for the first time the kinetics of oxygen release to the model wine.

11 Furthermore, the indirect contribution of oxygen to the ellagitannin disappearance by 12 boosting auto-oxidative reactions has also been pointed out. Vescalgin seems to be the 13 ellagitannin most affected by the initial oxygen levels.

15 Keywords: dissolved oxygen, ellagitannins, oak chips, oxygen consuming kinetics, oak 16 wood. 


\section{INTRODUCTION}

18 Oak barrels allow wine to receive small quantities of oxygen, which facilitates the 19 process of aging in barrels. Some published studies describe the evolution of dissolved oxygen (DO) in a model wine in contact with wood, measuring the DO decrease due to the fact that ellagitannins, some of the components that can be transferred from the wood, could consume the oxygen. ${ }^{1,2}$ Other papers analyze the transfer kinetics of the main ellagitannins of oak wood to aging wines. ${ }^{1-6}$ In addition, there is undoubtedly interest in discovering the amount of oxygen that wood contributes to the wine (autooxygenation) either by adding wood chips $^{7}$ or even by the staves when the barrel is filled with wine. ${ }^{8}$ All these studies supply very significant information regarding the wood, ellagitannins and oxygen interaction. However, no studies have tackled the three aspects simultaneously, i.e. evaluating the oxygen and the ellagitannins provided by the oak wood to the aging wine, the decrease in dissolved oxygen in the wine due to consumption by some of the compounds that the wood release to the wine and finally, the role of the ellagitannins supplied by the wood in the decrease in the dissolved oxygen present in the wine.

The scenario is complex as, when oak wood is added to a wine (oak chips), they begin to soak releasing to the wine the oxygen adhering to their surface. This oxygen is not considered oxygen from the wood since it is not trapped in the porosity of the wood itself. When the wood starts to be impregnated with the wine and the liquid begins to fill the void spaces in the wood (wood porosity), the air contained in the wood is displaced so it commences to soak first and to flood afterwards. The problem lies in the fact that when the wood enters into contact with the model wine, an extraction process of a series of hydro soluble compounds with a great oxygen consumption capacity (reductants) occurs and these are postulated as buffer compounds acting by limiting wine 
42 oxidation. ${ }^{9,10}$ Because of this, the measurement of dissolved oxygen present in the

43 model wine does not reflect the oxygen contributed by the wood, but rather only the 44 oxygen remaining after the oxygen interaction with the compounds released by the 45 wood, among which ellagitannins stand out. Therefore, it is important to take into 46 account the oxygen contained in the oak wood as an oxidizing agent of the compounds 47 released by the wood.

48 The objectives of this work are to evaluate the role of ellagitannins as oxygen49 consuming compounds and the importance of oak chips as natural micro-oxygenators. 50 For this, it is necessary to study the evolution of dissolved oxygen and the content of 51 ellagitannins in a model wine treated with oak chips. This paper presents, for the first 52 time, the results obtained on simultaneously evaluating the oxygen and the ellagitannins 53 contributed by French oak chips to a model wine in different scenarios: when the model 54 wine only has the oxygen provided by the oak wood (to evaluate the most normal 55 situation when treating wines with alternatives), when the model wine and the oak wood 56 are completely free from oxygen (in order to evaluate the ellagitannins content in a 57 completely oxygen-free scenario) and when wine has the maximum oxygen content 58 possible in a winey situation (air saturated medium).

59 MATERIALS AND METHODS

60 Wood. Medium toasted French oak chips (Quercus petraea (Matt.) Liebl.) supplied by 61 OenoWood International (Cognac, France) were used, with an average size of 1.2-1.5 62 cm long; 0.9-1.1 cm wide, 0.1-0.3 cm deep, $0.562 \mathrm{~g} / \mathrm{cm}^{3}$ density and a weight/surface 63 ratio of $0.1 \mathrm{~g} / \mathrm{cm}^{2}$. The porosity of the oak wood $(63.2 \%)$ was calculated as described 64 elsewhere. ${ }^{11}$ A medium level toasting process $\left(160-170^{\circ} \mathrm{C}\right.$ during $\left.20 \mathrm{~min}\right)$ was carried 65 out. The oak chips dose used was $10 \mathrm{~g} / \mathrm{L}$. 
66 Model wine. Hydroalcoholic solution (12.5\%) of $\mathrm{pH} 3.5$ was used. This solution has

67 been proved not to consume oxygen by measuring the DO consecutively in a 68 hermetically sealed container. All the tests were carried out in triplicate in 1.15 liter clear glass containers (DURAN Group GmbH, Germany) which, as they were endowed with butyl septum, maintained water/air tightness throughout the experiment (this was checked beforehand).

Experimental design. Different simultaneous tests were carried out providing comprehensive knowledge of the evolution of the dissolved oxygen and the ellagitannins released into the model wine stored with French oak chips for 120 days. Specifically, the DO and the ellagitannin total and individual (castalagin, vescalagin, grandinin and roburin E) contents were evaluated in a deoxygenated model wine with deoxygenated chips, that is free from oxygen (model system F), in a deoxygenated model wine with chips (model system $\mathbf{O W}$ ) and in a model wine saturated with air and with chips (model system OS). Finally, the increase in wood weight was evaluated when it was flooded by the model wine (impregnation test) (Figure 1). All the tests were carried out in triplicate. A detailed explanation of determination procedures of oxygen kinetics and wood impregnation can be read in Supporting Information.

Measurement of DO. The monitoring of DO was performed with integrated optical oxygen sensors in the transparent bottles closed with butyl septum to ensure no oxygen contamination. The sensors were spots of oxygen sensitive redflash indicators (PyroScience GmbH, Aachen, Germany) glued to the inner wall of the clear glass containers [resolution: $0.01 \% \mathrm{O}_{2}(0.005 \mathrm{mg} / \mathrm{L})$ at $1 \% \mathrm{O}_{2}, 0.05 \% \mathrm{O}_{2}(0.025 \mathrm{mg} / \mathrm{L})$ at $20 \% \mathrm{O}_{2}$; Accuracy: $\pm 0.02 \% \mathrm{O}_{2}(0.01 \mathrm{mg} / \mathrm{L})$ at $1 \% \mathrm{O}_{2}$ or $\pm 0.2 \% \mathrm{O}_{2}(0.1 \mathrm{mg} / \mathrm{L})$ at $20 \%$ $\mathrm{O}_{2}$ ], which allow DO readings by means of nine optical fibers connected to three FireSting $\mathrm{O}_{2}$ optical oxygen meters (PyroScience $\mathrm{GmbH}$, Aachen, Germany). The 
91 samples were kept at a constant room temperature of $15 \pm 0.5^{\circ} \mathrm{C}$. The oxygen sensors of 92 each bottle were calibrated according to the manufacturers' protocol, with 93 measurements performed at two calibration points: oxygen-free water $(0 \%$ air 94 saturation) and air-saturated water (100\% air saturation). Measurements were performed 95 in ultrapure water in saturation conditions according to ISO 5814:2012 (ISO, 2013) and 96 in oxygen-free water at a concentration of $0 \mathrm{mg} / \mathrm{L}$. The $0 \%$ calibration standard was 97 prepared based on a strong reductant; in this case, sodium dithionite $\left(\mathrm{Na}_{2} \mathrm{~S}_{2} \mathrm{O}_{4}\right)$ 98 (Panreac, Barcelona, Spain) at a concentration of $30 \mathrm{~g} / \mathrm{L}$.

99 All oxygen-measuring equipment had a temperature probe, pressure transducer and 100 humidity sensors used for temperature, pressure and humidity compensation. The 101 corresponding temperature probes were in contact with bottles, independently from the 102 luminescence equipment so as to have other means of correcting the measured values 103 and ensuring the quality of the measurements.

104 Phenolic composition of the oak chips. In order to study the total phenolic 105 composition, oak chips were ground and the powder was exhaustively extracted (5 106 extractions, $15 \mathrm{~min}$ of sonication per extraction) in triplicate with a solution of 107 methanol:water (50:50) previously sparged with Nitrogen. Extracts were concentrated in 108 a rotary vacuum evaporator and dissolved in ultrapure water. Samples were analyzed by 109 means of HPLC-DAD-MS ${ }^{\mathrm{n}}$-multiple reaction monitoring (MRM) analysis after the 110 addition of (-)-gallocatechin $(0.015 \mathrm{mg} / \mathrm{mL})$ as internal standard and filtration $(0.45 \mu \mathrm{m}$ 111 hydrophilic PVDF Clarinert ${ }^{\mathrm{TM}}$ Syring Filters, Agela Technologies, Wilmington, DE 112 19808, USA). This first preliminary study can supply information about the phenolic 113 potentiality of the wood employed in the present study. However, the extractability of 114 the phenolic compounds is influenced by the size of the wood piece from which they are 115 extracted. ${ }^{12}$ For this reason, the same extraction procedure and analysis methodology 
116 were directly applied to the oak chips to determine their phenolic profile, which is more

117 related to the extraction that would take place in the model systems of the present study

118 and which might be used as a reference for the extraction process in the model systems.

119 Analysis of the ellagitannins extracted from the oak chips in the model systems. An

120 aliquot of each of the triplicates of each model system was sampled at ten different

121 moments during the study period. Samples were diluted 1:1 with acidified water (acetic

122 acid, pH 3.5). Then, (-)-gallocatechin was added to the samples as internal standard

$123(0.015 \mathrm{mg} / \mathrm{mL})$ and samples were filtered $\left(0.45 \mu \mathrm{m}\right.$ hydrophilic PVDF Clarinert ${ }^{\mathrm{TM}}$

124 Syring Filters) before the HPLC-DAD-MS ${ }^{\mathrm{n}}-\mathrm{MRM}$ analyses.

HPLC-DAD-MS ${ }^{\mathbf{n}}$-MRM analysis. HPLC-DAD analyses were performed in a Hewlett-

Packard 1100 series LC (Agilent Technologies, Waldbronn, Germany) with a

previously developed HPLC method. ${ }^{13}$ MS detection was performed in an API 3200

Qtrap equipped with an ESI source and a triple-quadrupole linear ion trap mass analyser controlled by Analyst 5.1 software (Applied Biosystems, Darmstadt, Germany). Mass conditions have also been previously optimised and validated for the qualitative and quantitative analyses of oak ellagitannins. ${ }^{14}$ To be precise, the four main oak ellagitannins (castalagin, vescalagin, roburin $\mathrm{E}$ and grandinin) were quantified through a multiple reaction monitoring analysis (MRM) in negative mode. Castalin and vescalin were quantified as castalagin equivalents, from the peaks observed in the XIC (extracted ion chromatogram) at $\mathrm{m} / \mathrm{z} 631$ and corrected by the signal of the internal standard in the $\mathrm{XIC}$ at $\mathrm{m} / \mathrm{z}$ 305. The evolutions over time of ellagic acid and compounds related to the thermal degradation of lignin (coniferaldehyde and sinapaldehyde) were monitored from the peaks observed in the chromatogram recorded at $250 \mathrm{~nm}$.

Data modelling. Microsoft Excel 2013 (Redmond,WA, USA) and SOLVER function 
141 different processes following a first order kinetic each one. Fitting was done by non-

142 linear regression, minimizing the squared errors by using an iteration protocol based on

143 the robust and reliable generalized reduced gradient (GRG) method. The goodness of fit

144 of the models was assessed using determination coefficient $\left(\mathrm{R}^{2}\right)$. Default values were

145 randomly selected before starting the fitting and negative values were restricted during

146 fitting.

147 RESULTS AND DISCUSSION

148 Impregnation of the oak chips during wine aging.

149 The weight increase of the chips involves adding model wine to the wood: this addition 150 of liquid is in the form of bound water until the humidity of the wood reaches the Fiber 151 saturation point (FSP), which is $30 \%$ in oak (Figure 2a). The water is not bound above $15230 \%$ : it is known as free water and occupies the void space, which entails wood 153 porosity, displacing the air trapped in it, which contains $20.96 \%$ oxygen. Thus, the 154 oxygen transferred by the chips over aging time due to wood impregnation was 155 calculated by the increase in weight evaluated in the impregnation test. Figure $2 \mathrm{~b}$ shows 156 who estimated that the wood in the chips with a density of $0.645 \mathrm{~g} / \mathrm{cm}^{3}$ had a porosity of 
166 chips in water). These data ${ }^{7}$ were used to determine that, when the chips flooded with 167 the wine, they would provide $0.135 \mathrm{mg}$ oxygen per $\mathrm{g}$ of oak chips. A correct estimate of 168 the oxygen contained in alternative products (oak chips, staves, cubes...) which are 169 added to wine is very important in wine aging processes. The wine needs to count on the oxygen necessary to evolve appropriately during these processes of aging with wood

171 products, so the dose of oxygen provided by the wood itself needs to be added to that 172 added by active or passive micro-oxygenation. ${ }^{15}$

173 In addition, Figure $2 \mathrm{~b}$ shows the evolution of the measured DO present in the 174 deoxygenated model wine from the moment when it enters into contact with chips 175 (OW). The results indicate that the kinetics of oxygen transfer from the chips is greater 176 than the oxygen consumption kinetics in the first day of aging, because of which a 177 constant increase in DO content is observed in the model wine until that time. 178 Afterwards, and although the oxygen transferred from the chips increases due to its 179 impregnation, the DO reading in Figure $2 \mathrm{~b}$ shows that oxygen consumption by oak 180 compounds is taking place. The oxygen still grows until day 3 when oxygen consumption seems to be similar to oxygen release from oak, so that the DO content stabilizes (Figure 2b). From that moment the dissolved oxygen in the model wine begins to fall as a consequence of the slowdown in the transfer of oxygen from the wood and the consumption of the oxygen released. This consumption is high and leaves the model wine without oxygen in 55 days.

186 This result indicates that after 5 days the consumption of the oxygen transferred from 187 the chips by the substances released from wood (mainly ellagitannins) is clearly detectable and after 55 days there is practically no oxygen in the wine. This situation reproduces the normal scenario in an aging process of a finished red wine with oak chips and micro-oxygenation, in which the quantity of oxygen added to the wine in the 
191 first few weeks is exclusively that transferred from the wood chips with which it ages.

192 Afterwards, depending on the process, small quantities of oxygen are added using the 193 micro-oxygenation technique, which provides the oxygen required for the wine to 194 evolve appropriately. The dose varies according to the type of wine and the alternative 195 product used (size, type of wood...). ${ }^{15,16}$

196 Oxygen kinetics in the model systems.

197 It has been possible to adjust by least squares the evolution of the total DO in the model 198 system where the impregnation test was carried out (Figure 3a) and in model system 199 OW (Figure 3b) to a kinetic model that comprises the two main processes that have 200 been observed to occur in the present study: (1) oxygen release from oak wood $\left(\mathrm{O}_{2}\right.$ 201 released from wood) and (2) oxygen consumption $\left(\mathrm{C}_{\mathrm{con}}\right)$. Both processes can occur 202 simultaneously and it was assumed to follow a first order kinetics.

203 Regarding oxygen release from wood $\left(\mathrm{C}_{\mathrm{wood}}\right)$, it can be assimilated to two different sub204 processes, on one hand the oxygen adsorbed on the chips' surface together with the 205 oxygen released when the first mm thickness of the oak chips wets over $30 \% \mathrm{MC}\left(\mathrm{C}_{\mathrm{de}}\right)$.

206 On the other hand, the oxygen entrapped in the void space of the wood (porosity) when 207 it floods with the model wine and the trapped air is displaced outside of the wood $\left(\mathrm{C}_{\mathrm{fl}}\right)$ 208 (Figure 3a). Thus, the DO concentration released from oak wood could be calculated at 209 each moment by the following equation (Eqn.1):

$$
\mathrm{C}_{\mathrm{wood}}=\mathrm{C}_{\mathrm{de}} *\left(1-\mathrm{e}^{-K \mathrm{de} * \mathrm{t}}\right)+\mathrm{C}_{\mathrm{fl}} *\left(1-\mathrm{e}^{-K \mathrm{fl} * \mathrm{t}}\right)
$$

$211 \mathrm{C}_{\mathrm{de}}$ and $\mathrm{C}_{\mathrm{fl}}$ are the dissolved oxygen concentrations $(\mathrm{mg} / \mathrm{L})$ involved in each process 212 (de: desorption; fl: flood) and $k_{\mathrm{de}}$ and $k_{\mathrm{fl}}\left(\mathrm{day}^{-1}\right)$ are the kinetic constants of these 213 processes.

214 Figure 3a shows the adjustment of the model to the real data in the model system where 215 the impregnation test was carried out as well as the curves of the two oxygen release 
216 processes (desorption $\mathrm{C}_{\mathrm{de}}=1.712 ; k_{\mathrm{de}}=0.833$, and oxygen displaced by model wine

217 when flood $\left.\mathrm{C}_{\mathrm{fl}}=0.443 ; k_{\mathrm{fl}}=0.043\right)$ from oak wood shown separately. In all cases the 218 goodness of the adjustment is higher than 0.96 .

219 When modeling the real dissolved oxygen present in $\mathbf{O W}$ model wine it is necessary to adjust by least squares the evolution of the total DO content $(\mathrm{C})$ to a kinetic model that comprises the two main processes that have been observed to occur in the present study: the oxygen release from wood $\left(\mathrm{C}_{\text {wood }}\right)$ which has been described before, and the oxygen consumption $\left(\mathrm{C}_{\mathrm{con}}\right)$. Hence, the total $\mathrm{DO}$ content could be calculated at each moment by the following equation (Eqn.2):

$\mathrm{C}=\mathrm{C}_{\mathrm{wood}}-\mathrm{C}_{\text {con }} *\left(1-\mathrm{e}^{-K \operatorname{con} * t}\right)=\mathrm{C}_{\mathrm{de}} *\left(1-\mathrm{e}^{-K \mathrm{de}^{*} t}\right)+\mathrm{C}_{\mathrm{fl}} *\left(1-\mathrm{e}^{-K \mathrm{ff} * t}\right)-\mathrm{C}_{\operatorname{con}} *\left(1-\mathrm{e}^{-K \operatorname{con} * t}\right)$ $\mathrm{C}_{\text {con }}$ is the dissolved oxygen concentrations $(\mathrm{mg} / \mathrm{L})$ involved in consumption and $k_{\text {con }}$ $\left(\right.$ day $\left.^{-1}\right)$ is the kinetic constant of this process.

Figure $3 \mathrm{~b}$ shows the adjustment of the model to the real data in the $\mathbf{O W}$ model system as well as the curves of the oxygen release and the oxygen consumed by oak wood compounds $\left(\mathrm{C}_{\mathrm{con}}=2.18 ; k_{\mathrm{con}}=0.059\right)$, the goodness of the adjustment is higher than 0.99. Thus it has been possible to determine the kinetics of oxygen consumption by the compounds released by the wood.

Another model system in which liquid was saturated with air (OS) was also studied. This is an unusual situation during wine aging, but possible in a winery scenario whose study could help to understand the phenomena occurring when oxygen availability is at a maximum. From the results obtained and shown in Figure 3c, it can be deduced that the substances transferred from the wood chips gradually consume the oxygen available in the wine, thus producing an oxygen consumption kinetic which is described by the tendency shown in the figure. 
240 The substances transferred from the dosed wood $(10 \mathrm{~g} / \mathrm{L})$ to an air-saturated model wine

241 (OS) needed almost 4 months to exhaust the oxygen. In the model system OS, with air-

242 saturated model wine but also with the oxygen released from oak wood chips, the

243 oxygen release from the chips can be either considered or neglected. In this second case,

244 it can be considered that all the oxygen present in the model system is dissolved from

245 the beginning. In both cases the degradation would be similar, and the oxygen

246 concentration (C) in the model system OS could be calculated at each moment by the

247 following equation (Eqn.3):

$$
\mathrm{C}=\mathrm{C}_{\mathrm{ini}}-\mathrm{C}_{\text {con }} *\left(1-\mathrm{e}^{-K \operatorname{con} * t}\right)
$$

$249 \mathrm{C}_{\mathrm{ini}}$ is the initial oxygen concentration in the model system $\mathrm{OS}\left(\mathrm{C}_{\mathrm{ini}}=10.86 \mathrm{mg} / \mathrm{L}\right), \mathrm{C}_{\text {con }}$

250 is the oxygen consumed $(\mathrm{mg} / \mathrm{L})$ and $k_{\text {con }}\left(\right.$ day $\left.^{-1}\right)$ is the kinetic constant of this 251 consumption process $\left(\mathrm{C}_{\mathrm{con}}=10.86 ; k_{\mathrm{con}}=0.03\right)$. The goodness of the adjustment is 252 higher than 0.99 . Furthermore, the kinetics of that consuming process is very slow in 253 relation to that observed in model $\mathbf{O W}$, which can be related to the higher DO content 254 in model system OS in relation to $\mathbf{O W}$ (up to five times higher than the oxygen 255 potentially released from wood in model system $\mathbf{O W}$ ).

256 Evolution of oak ellagitannins and related compounds in the model systems.

257 The preliminary studies carried out in the oak chips powder and directly in the oak chips 258 (Supporting Information section 1) revealed the presence of ellagitannins and 259 ellagitannin-related compounds, ellagic acid and compounds related to the thermal 260 degradation of lignin, ${ }^{17}$ such as coniferaldehyde and sinapaldehyde. The ellagitannins 261 and ellagitannin-related compounds constituted the majority of the extractable 262 compounds, accounting for almost $80 \%$ of the total area of the chromatogram of the oak 263 chips extracts recorded at $250 \mathrm{~nm}$. Bearing this fact in mind and taking into account that 264 they can take part in oxidation reactions, ${ }^{18}$ it might be hypothesized that ellagitannins 
265

266

267

268

269

270

271

272

273

274

275

276

277

could be the main oxygen consumers in the model systems. In order to verify this hypothesis, the evolutions of the levels of these compounds were monitored along all the study in all the three model systems.

As is mentioned above model system $\mathbf{F}$ will serve as the reference for the behavior of the ellagitannins in absence of oxygen. In this system, dissolved oxygen content was close to zero (values below $0.01 \mathrm{mg} / \mathrm{L}$ ) throughout the test. Thus, differences in the evolution of the ellagitannins in model systems $\mathbf{O W}$ and $\mathbf{O S}$ in relation to model system F could be attributed to the presence of different levels of oxygen.

Figure 4 shows the evolution of the total ellagitannin content in the three model systems. These total ellagitannin contents were calculated as the sum of the four main oak ellagitannins detected in the oak chips (castalagin, vescalagin, grandinin and roburin E). Castalin and vescalin, which were also present in the oak chips but can be formed from the other ellagitannins, were monitored separately in order to evaluate the involvement of oxygen in their formation during the experiment.

In model system $\mathbf{F}$, the evolution of the ellagitannins can be divided into three steps: first, a fast increase of the levels followed by a somehow stabilization and then, by a decrease at two different rates. In that model system, it can be deduced that the only processes that are taking place during the first stage are the extraction of the ellagitannins from the oak chips and their diffusion to the model solution. First, the evolution until the moment of the maximum content (Figure 4, Inset) is in accordance with the two-steps kinetic model recently proposed by García-Estévez and co-workers $(2015)^{3}$ to explain the extraction of ellagitannins from wood. Thus, at the very beginning of the experiment, ellagitannins were already detectable $(52 \mathrm{mg} / \mathrm{L})$ in model system F, which might correspond to the amounts extracted during the washing step (extraction of the ellagitannins from the surface of the chips). Then, the levels increased 
until day eight (the day when the maximum content was reached) but at two different rates, one faster until the fifth day and the other slower from that day to the eighth day, which fits well with the diffusion process at two different rates proposed by the kinetic model. Second, the maximum content of total ellagitannins reached in this model system $(627.6 \mathrm{mg} / \mathrm{L})$ was almost the same as that detected in the preliminary study on the exhaustive extraction of the oak chips $(637.9 \mathrm{mg} / \mathrm{L})$ with methanol:water (50:50). The second stage ranged from day 8 to day 17 and corresponded to a somehow "plateau" (above all in the case of castalagin, as it will be shown below) or slow decrease of the levels. At this stage, the extraction and disappearance of the ellagitannins were quite balanced. However, during the last stage of the ellagitannin evolution in model system $\mathbf{F}$, the disappearance rate increased, which was due to fact that the reactions leading to the disappearance of the oak native ellagitannins were more important than their extraction from the oak chips. Since in that model system oxygen was absent, the ellagitannins themselves can be at the origin of their disappearance probably through auto-oxidation reactions (from now onwards "oxygen-independent" reactions). Despite this decrease in the total ellagitannin content, at the end of the study (more than three months) $60 \%$ of the maximum content was still detectable in the model system.

Respecting the evolution of the total ellagitannin content in model system $\mathbf{O W}$, it can be seen in Figure 4 that the "plateau" stage is absent and only the increase and decrease phases can be observed. Regarding the increase stage, the maximum level is lower and is reached earlier (day 6) than in model system F. Since the chips employed in both model systems are the same as well as the model solution, the only difference between model systems $\mathbf{F}$ and $\mathbf{O W}$ is the absence of oxygen in the former and the availability of the oxygen trapped into the chips in the latter one. In model system $\mathbf{O W}$, two processes 
315 are occurring simultaneously when the oak chips are getting wet: the extraction of the 316 ellagitannins and the dissolution of the oxygen adsorbed in the surface of the oak chips and of that released from the first $\mathrm{mm}$ thickness. Consequently, ellagitannins can encounter oxygen in the solution from the very beginning and react with it, causing a decrease in the levels of both types of compounds. As it was mentioned above and shown in Figure 2, the maximum DO level determined in model system $\mathbf{O W}$ was quite lower than the theoretical oxygen level that should be reached if the release from oak chips were the only process occurring in the model system. This can be interpreted as a rapid consumption of part of the oxygen released from the oak chips. Thus, ellagitannins, the only compounds present in the solution from the very beginning, might be the main consumers of this oxygen and this would explain the lower levels at the maximum. Furthermore, the absence of the "plateau" phase and the earlier beginning of the decrease phase in model system $\mathbf{O W}$ in relation to model system $\mathbf{F}$ can oxygen itself can also can boost the oxygen-independent reactions through the rates were observed when there was more oxygen available (from day 6 to 20), decreasing then from day 20 to day 40, as the availability of the oxygen decreases (from 
340 experiment, when the already reduced oxygen levels fell until disappearance, the rate

341 was similar to that observed in model system $\mathbf{F}$, which is indicative of a reduction of the

342 influence of oxygen-dependent reactions in model system $\mathbf{O W}$ during this period.

343 Respecting model system OS, the evolution of the ellagitannins was similar to that

344 observed in model system $\mathbf{O W}$, with a first stage where the levels increased, reached a

345 maximum at day 5 and then decreased. As it could be expected from the different levels

346 of dissolved oxygen detected in them, the ellagitannin contents were lower in all the

347 stages in model system OS. However, differences were lower than expected if we take

348 into account that the dissolved oxygen detected in model system $\mathbf{O W}$ represented 349 during most of the experiment less than $10 \%$ of the dissolved oxygen detected in model 350 system OS. For example, at the moment of maximum ellagitannin levels the total 351 medium content was $627.6 \mathrm{mg} / \mathrm{L}, 495.7 \mathrm{mg} / \mathrm{L}$ and $393.4 \mathrm{mg} / \mathrm{L}$ for model systems $\mathbf{F}$, 352 OW and $\mathbf{O S}$, respectively, whereas the DO determined were $0 \mathrm{mg} / \mathrm{L}, 1.19 \mathrm{mg} / \mathrm{L}$ and $3539.23 \mathrm{mg} / \mathrm{L}$, respectively. Thus, the increase from $0 \mathrm{mg} / \mathrm{L}$ to $1.19 \mathrm{mg} / \mathrm{L}$ caused a 354 reduction of $21 \%$ of the total content in model system $\mathbf{O W}$ in relation to $\mathbf{F}$, whereas an 355 additional increase of $8 \mathrm{mg} / \mathrm{L}$ in model system $\mathbf{O S}$ in relation to $\mathbf{O W}$ only caused an 356 additional decrease of $21 \%$ of the total content despite the greater increase in the oxygen 357 levels. As it was previously indicated for model system $\mathbf{O W}$, the initial moment when 358 both the ellagitannins and the oxygen are extracted from the oak chips to the model 359 solution seem to be a crucial step. At this moment, the oxygen-to-ellagitannins ratio has 360 to be very similar in both model systems (the oak chips are the same in both cases and 361 consequently, the ellagitannin contents and the oxygen trapped in them) and it has to be 362 probably much higher than later on, when ellagitannins and oxygen are diffusing to the 363 model solution. Thus, the oxygen-dependent reactions will start in both model systems 364 simultaneously and the small differences in their ellagitannin levels might be explained 
by the differences in the oxygen present in the model solution of model system OS. Furthermore, the presence of oxygen in the model solution can decrease, in turn, the rate of oxygen release from the chips, since the concentration gradient is smaller than in the case of model system OW. This hypothesis would explain why the ellagitannin levels are not so different in both model systems during the increase phase despite the differences in the DO contents. On the contrary, during the decrease phase the higher oxygen levels in model system OS would affect the ellagitannin levels since ellagitannins encounter more oxygen molecules during their diffusion to the solution than in model system $\mathbf{O W}$, where oxygen was initially absent in the solvent.

The influence of the higher levels of dissolved oxygen in model system OS can be more clearly observed in the fastest stage of the decrease phase (Figure 4), which lasted 35 days in that model system and only 15 days in model system OW. Furthermore, the slope of this fastest stage is higher in model system OS than in model system OW. In addition to the higher direct effect of oxygen on the ellagitannin levels, the higher oxygen levels have probably promoted the oxygen-independent reactions, thus contributing to the greater disappearance of ellagitannins in model system OS during all the experiment. Nevertheless, the differences between ellagitannin levels were not, again, as great as it could be expected from the differences in the DO. It seems that the influence on the ellagitannin levels of the oxygen released from the chips is higher than the influence of the oxygen present in the model solution.

It is also important to remark that from day 40 to the end of the study the slope of the decrease is the same in both model systems and very similar to that observed in model system F, despite the relatively high DO levels in model system OS (circa $3.5 \mathrm{mg} / \mathrm{L}$ ) and the almost absent oxygen levels in model system $\mathbf{O W}$. As indicated for model system $\mathbf{O W}$, the decrease observed from day 40 to the end is mainly governed by the 
same oxygen-independent reactions that occurred in model system $\mathbf{F}$, although in the case of model OS there is oxygen still available. This fact points to a small direct influence of this still available oxygen on the ellagitannin levels at this late stage of the experiment. However, oxygen continues disappearing from day 40 to the end, probably by taking part in reactions with the products of the oxygen-independent reactions.

It has been possible to adjust by least squares the evolution of the total ellagitannin content in the three different model systems to a kinetic model that comprises the three main processes that have been observed to occur in the present study: extraction (1), oxygen-dependent reactions (2) and oxygen-independent reactions and/or degradation (3). The three processes can occur simultaneously and it was assumed to follow a first order kinetics. According to the proposed model, the ellagitannin concentration could be calculated at each moment by the following equation (Eqn.4):

$$
[\text { Elag }]=\mathrm{C}_{\mathrm{ext}} *\left(1-\mathrm{e}^{-K e x t^{*} t}\right)-\mathrm{C}_{\mathrm{ox}} *\left(1-\mathrm{e}^{-K o x^{*} t}\right)-\mathrm{C}_{\mathrm{deg}} *\left(1-\mathrm{e}^{-K d e g^{*} t}\right) \quad \text { [Eqn.4] }
$$

$\mathrm{C}_{e x t}, \mathrm{C}_{o x}$ and $\mathrm{C}_{d e g}$ are the ellagitannin concentrations $(\mathrm{mg} / \mathrm{L})$ involved in each process (ext: extraction; ox: oxygen-dependent reactions; deg: oxygen-independent reactions) and $k_{\text {ext }}, k_{\text {ox }}$ and $k_{\text {deg }}\left(\right.$ day $\left.^{-1}\right)$ are the kinetic constants of these processes.

The fitting of the ellagitannin evolution of the three model systems studied in this work was performed at the same time, using randomly-selected starting values. Figure 5 shows the adjustment of the model to the real data in the three model system as well as the curves of the three processes described by the model separately. In all cases the goodness of the adjustment is higher than 0.99. Table 1 shows the values of the constants and of the theoretical maximum concentrations of ellagitannins involved in each process. Concerning the oxygen-dependent reactions it can clearly be seen that the maximum ellagitannin concentration susceptible of disappearing as a consequence of them is different in the different model systems. In model system $\mathbf{F}$, where the oxygen 
415 is absent, this process is practically irrelevant in contrast to circa 270 and $350 \mathrm{mg} / \mathrm{L}$

416 susceptible to disappear as a consequence of this process in model systems $\mathbf{O W}$ and

417 OS, respectively. Between model systems OW and OS there were also differences: as

418 expected, $\mathrm{C}_{o x}$ and $k_{o x}$ were higher in the latter, which is in accordance with the dissolved

419 oxygen levels. However, as previously commented, these differences were lower than it

420 could be expected from the differences in the dissolved oxygen levels, which can be

421 pointing out to the relevance of the oxygen trapped in the oak chips. Respecting the two

422 types of reactions leading to the disappearance of the ellagitannins, the kinetics of the

423 oxygen-independent reactions were slower than the kinetics of the oxygen-dependent

424 reactions in all the model systems. Moreover, whereas in model system F,

425 disappearance of the ellagitannins were almost exclusively due to oxygen-independent

426 reactions, in model systems $\mathbf{O W}$ and $\mathbf{O S}$ both the amount of ellagitannin that disappear

427 and the rate of the disappearance due to both processes increased as the dissolved

428 oxygen levels increased. This can be indicating again that the oxygen-independent

429 reactions can be favored by the products of the oxygen-dependent reactions.

430 Individual ellagitannins. The evolutions of the four main ellagitannins were also 431 studied individually in the three model systems (Figure 6). They all showed the same 432 stages as those observed for the total ellagitannin content. However, differences among 433 the different compounds were detected mainly concerning the two major ellagitannins, 434 castalagin and vescalagin, and their reactivity towards oxygen. The initial levels of 435 vescalagin seem to be clearly affected by the presence of oxygen, since the maximum 436 content was reduced in $20 \%$ and $45 \%$ in model systems OW and OS, respectively, in 437 relation to $\mathbf{F}$. On the contrary, the maximum content of castalagin was less reduced: $43815 \%$ in $\mathbf{O W}$ and only $22 \%$ in $\mathbf{O S}$ in relation to that determined in $\mathbf{F}$. This could indicate 439 that vescalagin is the ellagitannin most involved in reactions with oxygen at the 
440 beginning, maybe partly due to its higher trend to be extracted from chips during the

441 washing step. ${ }^{3}$ However, the additional oxygen content existing in models system $\mathbf{O S}$ in

442 relation to $\mathbf{O W}$ hardly affected the rate of the first part of the decreasing phase in the

443 case of vescalagin, but provoked higher rates in the case of castalagin. This might be

444 indicative of a different behavior of castalagin and vescalagin towards oxygen, which

445 might be attributed to the different configuration of $\mathrm{C} 1$ (hydroxyl group in $\beta$

446 configuration for vescalagin and in $\alpha$ for castalagin) as occurs for other types of

447 reactions. $^{19,20}$

448 The present study has also confirmed the higher reactivity that is usually attributed to 449 vescalagin in relation to castalagin. ${ }^{19,20}$ In fact, in model system $\mathbf{F}$, where oxygen is 450 absent, vescalagin started the decrease phase much early than castalagin and at the end 451 of the study, $33 \%$ of the maximum content of castalagin was reduced whereas the levels 452 of vescalagin decreased $47 \%$.

453 Respecting grandinin and roburin E, they showed similar evolutions, which were more 454 similar to that of vescalagin than to that of castalagin. This might be related to the 455 configuration of $\mathrm{C} 1$, which is the same for these three compounds and different for 456 castalagin. On the basis of the important losses from the maximum content observed in 457 model system $\mathbf{F}$, it can be deduced that the oxygen-independent reactions are quite 458 relevant for grandinin and roburin E. Moreover, the presence of oxygen also caused 459 important reduction of the maximum content (about $40 \%$ in OW and 55\% in OS), thus 460 indicating that grandinin and roburin $\mathrm{E}$ are more sensitive than vescalagin and 461 castalagin to both oxygen-independent and oxygen-dependent reactions.

462 The kinetic model proposed for the total ellagitannin content was also applied to the 463 individual contents in order to evaluate the relevance of the three processes in the 464 evolution of the different ellagitannins (Supporting Information Table S2). For 
465

466

467

comparative purposes among the different ellagitannins, the ratio between the values of $\mathrm{C}_{o x}$ and $\mathrm{C}_{d e g}$ and that of $\mathrm{C}_{e x t}$ were calculated for each compound in each model system. In model system $\mathbf{F}$, where oxygen-dependent reactions were absent, roburin $\mathrm{E}$ was the ellagitannin most affected by oxygen-independent reactions, followed by grandinin and vescalagin. Castalagin, on the contrary, showed higher stability towards this type of reactions. The increase of DO levels from model system $\mathbf{F}$ to $\mathbf{O W}$ and from $\mathbf{O W}$ to $\mathbf{O S}$ caused an increase in $\mathrm{C}_{o x}$ in all the ellagitannins, thus corroborating again the influence of the oxygen in the evolution of the ellagitannins.

Evolution of castalin and vescalin. The evolution of castalin and vescalin, which were already detected in the oak extracts, remind to an extraction process but their contents seem to be also conditioned by the oxygen levels, since important differences were observed among model systems (Supporting Information Figure S2). During all the study, model system $\mathbf{O S}$ showed the highest contents, followed by $\mathbf{O W}$ and $\mathbf{F}$. At the end of the study, the levels of castalin were $c a .1 .7$ and 2.5 -fold higher in the model systems OW and $\mathbf{O S}$, respectively, than in model system F, whereas the content of vescalin was $c a$. 1.5 and 3-fold higher in model systems $\mathbf{O W}$ and $\mathbf{O S}$ than in F. Thus, it seems that castalin and vescalin could be formed during the experiment and that their levels depend on the levels of oxygen. This influence of oxygen might be either direct, if the oxygen were the agent promoting the hydrolysis reactions among the ellagitannins or indirect, if the agent were the oxidation products of the ellagitannins.

Moreover, the formation of vescalin seems to be more affected by the oxygen levels than the formation of castalin, above all during the first stages. Thus, in absence of oxygen (F), the percentages of castalin and vescalin evolved from 97:3 at the first day to 69:31 at the end of the study. In model systems OW and OS, this ratio was, respectively, 70:30 and 60:40 after 24 hours and it remains quite stable during all the 
490 study. This fact confirms the correlation of the greater disappearance of vescalagin as

491 the oxygen levels increase and the greater formation of hydrolysis products, pointing

492

493

494

495

496

497

out again to the possible involvement of oxygen in the hydrolysis reactions of the ellagitannins. Moreover, due to the large number of hydroxyl groups in their structure, these hydrolysis products may react, in turn, with oxygen, which would partly explain the trend to stabilization observed in model systems $\mathbf{O S}$ and $\mathbf{O W}$, respectively, despite the decrease observed for the parent compounds during this same period.

Evolution of other compounds extracted from oak chips. Ellagic acid (Supporting Information Figure S3) can be formed after hydrolysis of the ellagitannins but it was also already present in the oak chips. For this reason, during the first five days the levels of ellagic acid increased in all the model system as a consequence of the extraction from the chips. From this point onwards, although a slight increasing trend could be observed, oscillation in the levels occurred in all the model systems. Furthermore, and unlike ellagitannins and castalin and vescalin, the evolution and the levels of ellagic acid were hardly affected by the presence of the oxygen trapped in the oak chips. Only in model system OS slightly higher contents could be observed, which could be pointing out to a higher involvement of the dissolved oxygen than the oxygen released from the chips in the formation of ellagic acid. However, from these results it seems that ellagic acid would not be a relevant oxygen consumer.

The possible role as oxygen consumers of other non-ellagitannin compounds extracted from wood, such as coniferaldehyde and sinapaldehyde, has been also evaluated. These compounds showed similar evolutions, and there are no differences between the different model systems during the first 20 days. Thus, it can be deduced that the evolution of these compounds was not directly influenced by the DO levels. However, at the end of the study, the contents of coniferaldehyde and sinapaldehyde were, 
515 respectively, 1.4- and 1.2-fold higher in model systems $\mathbf{O W}$ and $\mathbf{O S}$ than in $\mathbf{F}$, due to an 516 increase at fastest rates in the oxygen-containing model systems than in $\mathbf{F}$ and faster in 517 OS than in OW. This could point out an indirect involvement of oxygen in the 518 formation of these compounds, that is, it might be favored by the oxidation products of 519 the ellagitannins. Nevertheless, the levels seemed to be higher for model system OW 520 than for OS, which might be due to the simultaneous formation and subsequent loss of 521 these aldehydes by oxidative reactions in OS due to the high levels of oxygen. In fact, a 522 great reactivity of coniferaldehyde towards oxidants has been reported. ${ }^{21}$ In addition, the 523 possibility of the formation of the benzoic aldehydes from these cinnamic aldehydes by 524 chemical oxidation in hydroalcoholic medium has already reported. ${ }^{17}$

525 In summary, this study reports, for the first time, the kinetics of oxygen release from 526 French oak chips, comprising two main processes at different rates: a) the desorption of 527 the oxygen adsorbed in the surface of the chips and that trapped in the first $\mathrm{mm}$ of 528 thickness of the chips and b) the release of the oxygen entrapped in the void space of the 529 wood. Furthermore, this study is the first report on the kinetics of oxygen consumption 530 by oak components at two different levels of dissolved oxygen (oxygen released from 531 the oak chips in a deoxygenated model wine and in an air saturated model wine). The 532 study of the phenolic composition of the oak chips has revealed a clear quantitative 533 predominance of ellagitannins and ellagitannin-related compounds. In addition the 534 evolutions of their levels in absence and in presence of oxygen have confirmed their 535 role as the main oxygen consumers among the phenolic compounds released by oak 536 chips. The maximum ellagitannin levels were reduced in presence of oxygen and in a 537 greater extent when the oxygen levels were higher. However, the differences were not 538 as great as they might be expected from the different oxygen contents. This fact has 539 highlighted the relevance of the oxygen that is released from the oak chips on the 
540 evolution of the ellagitannins. Furthermore, this study has also underlined the relevance

541 of oxygen-independent reactions (related to the auto-oxidation of the ellagitannins) in

542 the evolution of the ellagitannin levels. These reactions were the main cause of the

543 decrease observed in the oxygen-free model system and were also important in the

544 oxygen-containing model systems, clearly observable when the levels of oxygen were

545 reduced. This study also reports, for the first time the influence of oxygen in the

546 formation of castalin and vescalin. From all these results it can be concluded that

547 although oxygen is not the only agent responsible for the disappearance of the

548 ellagitannins, the oxygen amounts supplied by the oak woods chips to a wine during an

549 alternative aging process can play a relevant role in consuming the ellagitannins

550 provided by the wood or in boosting their auto-oxidative reactions, thus affecting the

551 final levels of ellagitannins in the wine.

552 Acknowledgements

553 The authors express great appreciation to OenoWood International (Cognac, France) for

554 their collaboration.

555 Funding

556 This work was financed by the Ministerio de Ciencia e Innovación and FEDER funds

557 (AGL2014-54602-P and AGL2014-58486-C02-R-1) and by Junta de Castilla y León

558 (VA124U14 and VA028U16) of Spain.

559 Notes

560 The authors declare no competing financial interest.

561 Associated content

562 Supporting Information

563 1. Determination of the transfer and consumption kinetics of the oxygen contained in

564 the wood (OW). 
565 2. Determination of the evolution of dissolved oxygen in deoxygenated model wine and 566 deoxygenated chips (F).

567 3. Determination of the oxygen consumption kinetics by the substances transferred from 568 the wood (OS).

569 4. Determination of the oak chips impregnation kinetics (impregnation test).

570 5. Phenolic composition of the oak chips.

571 Table S1. Chromatographic, UV and mass spectral features and fragmentation patterns 572 of the compounds detected in the chromatogram recorded at $250 \mathrm{~nm}$.

573 Table S2. Parameters of the kinetic model proposed for the evolution of the individual 574 ellagitannins in the three model systems.

575 Figure S1. Chromatograms of the extracts made from the oak chips powder (a) and 576 directly from the oak chips (b) recorded at $250 \mathrm{~nm}$. The identity of the peaks is 577 indicated in Table S1.

578 Figure S2. Evolution of the levels (equivalents of castalagin, $\mathrm{mg} / \mathrm{L}$ ) of castalin (a) and 579 vescalin (b) in model systems F (green), OW (brown) and OS (red) during all the 580 experiment. Different lower-case letters indicate significant differences $(p<0.05)$ among 581 the different model systems at the same sampling point.

582 Figure S3. Evolution of the chromatographic area $(250 \mathrm{~nm})$ over time of Ellagic acid, 583 Coniferaldehyde and Sinapaldehyde in model systems F, OW and OS. Only differences 584 between the different model systems at the same sampling points were indicated when 585 significant $(p<0.05)$ by lower-case letters. 
587

588

589

590

591

592

593

594

595

596

597

598

599

600

601

602

603

604

605

606

607

608

609

610

611

\section{References}

(1) Navarro, M.; Kontoudakis, N.; Giordanengo, T.; Gómez-Alonso, S.; GarcíaRomero, E.; Fort, F.; Canals, J. M.; Hermosín-Gutíerrez, I.; Zamora, F. Oxygen consumption by oak chips in a model wine solution; Influence of the botanical origin, toast level and ellagitannin content. Food Chem. 2016, 199, 822-827.

(2) Pascual, O.; Vignault, A.; Gombau, J.; Navarro, M.; Gómez-Alonso, S.; GarcíaRomero, E.; Canals, J. M.; Hermosín-Gutíerrez, I.; Teissedre, P.-L.; Zamora, F. Oxygen consumption rates by different oenological tannins in a model wine solution. Food Chem. 2017. 234, 26-32.

(3) García-Estévez, I.; Alcalde-Eon, C.; Le Grottaglie, L.; Rivas-Gonzalo, J. C.; Escribano-Bailón, M. T. Understanding the ellagitannin extraction process from oak wood. Tetrahedron 2015, 71, 3089-3094.

(4) Masson, G.; Moutounet, M.; Puech, J. L. Ellagitannin Content of Oak Wood as a Function of Species and of Sampling Position in the Tree. Am. J. Enol. Vitic. 1995, 46, 262-268.

(5) Marinov, M. G.; Dimitrova, E. D.; Puech, J. L. Kinetics of ellagitannin extraction from oak wood using white wine. J. Wine Res. 1997, 8, 29-40.

(6) Masson, E.; Puech, J. L. The incidence of different modes of kiln drying on ellagitannin and volatile compound content of sessile oak, (*Quercus petraea* (Matt.) Liebl.). J. des Sci. Tech. la Tonnellerie 2000, 6, 59-78.

(7) Piracci, A.; Bucelli, P.; Faviere, V.; Giannetti, F.; Lo Scalzo, R.; Novello, E. Frammenti legnosi e vino: alcune specifiche tecniche di chips e staves di rovere. L'Enologo 2001, 7-8, 97-106.

(8) Qiu, Y. Phénomènes de transfert d'oxygène à travers la barrique, Université de Bordeaux, 2015. 
612 (9) Singleton, V. L. Maturation of Wines and Spirits - Comparisons, Facts, and Hypotheses. Am. J. Enol. Vitic. 1995, 46, 98-115.

(10) Vivas, N. Nonier, M.F. Vivas de Gaulejac, N. Reactions and occurrence of wine polyphenol evolution during the oxidative process in oak. In Proceedings of the twelfth Australian wine industry technical conference; 24-29 July 2004; Melbourne, VIC; Australian Wine Industry Technical Conference Inc.: Adelaide, SA: Adelaide, SA, 2005; pp 205-214.

(11) del Álamo-Sanza, M.; Cárcel, L. M.; Nevares, I. Characterization of the Oxygen Transmission Rate of Oak Wood Species Used in Cooperage. J. Agric. Food Chem. 2017, 65, 648-655.

(12) Fernández de Simón, B.; Cadahía, E.; del Álamo, M.; Nevares, I. Effect of size, seasoning and toasting in the volatile compounds in toasted oak wood and in a red wine treated with them. Anal. Chim. Acta 2010, 660, 211-220.

(13) García-Estévez, I.; Escribano-Bailón, M. T.; Rivas-Gonzalo, J. C.; Alcalde-Eon, C. Development of a fractionation method for the detection and identification of oak ellagitannins in red wines. Anal. Chim. Acta 2010, 660, 171-176.

(14) García-Estévez, I.; Escribano-Bailón, M. T.; Rivas-Gonzalo, J. C.; Alcalde-Eon, C. Validation of a mass spectrometry method to quantify oak ellagitannins in wine samples. J. Agric. Food Chem. 2012, 60, 1373-1379.

(15) Nevares, I.; del Álamo-Sanza, M. Wine Aging Technologies. In Recent advances in wine stabilization and conservation technologies; Jordão, A. M., Cosme, F., Eds.; Nova Science Publishers, Inc.: New York, 2016; pp 209-245.

(16) del Álamo, M.; Nevares, I.; Gallego, L.; Fernández de Simón, B.; Cadahía, E. Micro-oxygenation strategy depends on origin and size of oak chips or staves during accelerated red wine aging. Anal. Chim. Acta 2010, 660, 92-101. 
637 (17) Sarni, F.; Moutounet, M.; Puech, J. L.; Rabier, P. Effect of heat treatment of oak

638

639

640

641

642

643

644

645

646

647

648

649

650

651

652 wood extractable compounds. Holzforschung 1990, 44, 461-466.

(18) Vivas, N.; Glories, Y. Role of Oak Wood Ellagitannins in the Oxidation Process of Red Wines During Aging. Am. J. Enol. Vitic. 1996, 47, 103-107.

(19) Quideau, S.; Jourdes, M.; Lefeuvre, D.; Montaudon, D.; Saucier, C.; Glories, Y.; Pardon, P.; Pourquier, P. The chemistry of wine polyphenolic C-glycosidic ellagitannins targeting human topoisomerase II. Chem. - A Eur. J. 2005, 11, $6503-6513$.

(20) Jourdes, M.; Lefeuvre, D.; Quideau, S. C-Glycosidic Ellagitannins and Their Influence on Wine Chemistry. In Chemistry and biology of ellagitannins : an underestimated class of bioactive plant polyphenols; Quideau, S., Ed.; World Scientific: Singapore; Hackensack, NJ, 2009; pp 320-365.

(21) Pan, G. X.; Spencer, L.; Leary, G. J. Reactivity of ferulic acid and its derivatives toward hydrogen peroxide and peracetic acid. J. Agric. Food Chem. 1999, 47, $3325-3331$. 


\section{$653 \quad$ Figure captions}

654 Figure 1. Experimental design. Deoxygenated model wine with chips $(\mathbf{O W})$ or with 655 deoxygenated chips (F), model wine saturated with air and with chips (OS) and test of 656 impregnation of chips (impregnation test).

657 Figure 2. Increased weight of the chips over the time spent in the model wine (a) and 658 Evolution of the dissolved oxygen in model system OW (DO content) together with the 659 calculated oxygen amount released from wood (O2 released from wood). (b).

660 Figure 3. Evolution of the oxygen calculated as being transferred from the chips on 661 impregnating the model wine (model O2 from wood), and the oxygen desorption (Cde 662 model), flood (Cfl model) and total oxygen release from wood models (Cwood) (a) 663 Evolution of the consumed oxygen (Ccon model) calculated as difference between 664 oxygen release from wood and dissolved oxygen content (b) and evolution of the 665 oxygen consumption kinetics during the aging of air-saturated model wine in the 666 presence of oak chips (Model system OS) (c).

667 Figure 4. Evolution of the total ellagitannin contents $(\mathrm{mg} / \mathrm{L})$ in model systems $\mathbf{F}$ 668 (green), OW (brown) and OS (red) during all the experiment. The inset shows a detail 669 of these evolutions from day 0 to day 13.

670 Figure 5. Adjustment of the kinetic model to real data in model systems F (a), OW (b) 671 and OS (c). The curves of the three processes described by the kinetic model 672 (extraction, oxygen-dependent and oxygen-independent reactions) are also shown 673 separately for each model system.

674 Figure 6. Evolution of the individual ellagitannin contents $(\mathrm{mg} / \mathrm{L})($ castalagin, a;

675 vescalagin, b; grandinin, c; roburin E, d) in model systems F (green), OW (brown) and 676 OS (red) during all the experiment. 
Table 1. Values of the constants and of the theoretical maximum concentrations involved in each process in model systems $\mathbf{F}, \mathbf{O W}, \mathbf{O S}$.

\begin{tabular}{cccc}
\hline Total Ellagitannins & F & OW & OS \\
\hline $\mathrm{C}_{\mathrm{ext}}$ & 777.01 & 776.71 & 777.76 \\
$k_{\mathrm{ext}}$ & 0.328 & 0.465 & 0.467 \\
\hline $\mathrm{C}_{\mathrm{ox}}$ & 0.10 & 268.81 & 354.86 \\
$k_{\mathrm{ox}}$ & 0.014 & 0.180 & 0.225 \\
\hline $\mathrm{C}_{\mathrm{deg}}$ & 434.71 & 330.51 & 371.79 \\
$k_{\mathrm{deg}}$ & 0.028 & 0.033 & 0.040 \\
\hline
\end{tabular}

$\mathrm{C}(\mathrm{mg} / \mathrm{L}) ; k(1 /$ day $)$ 


\section{Figure 1.}

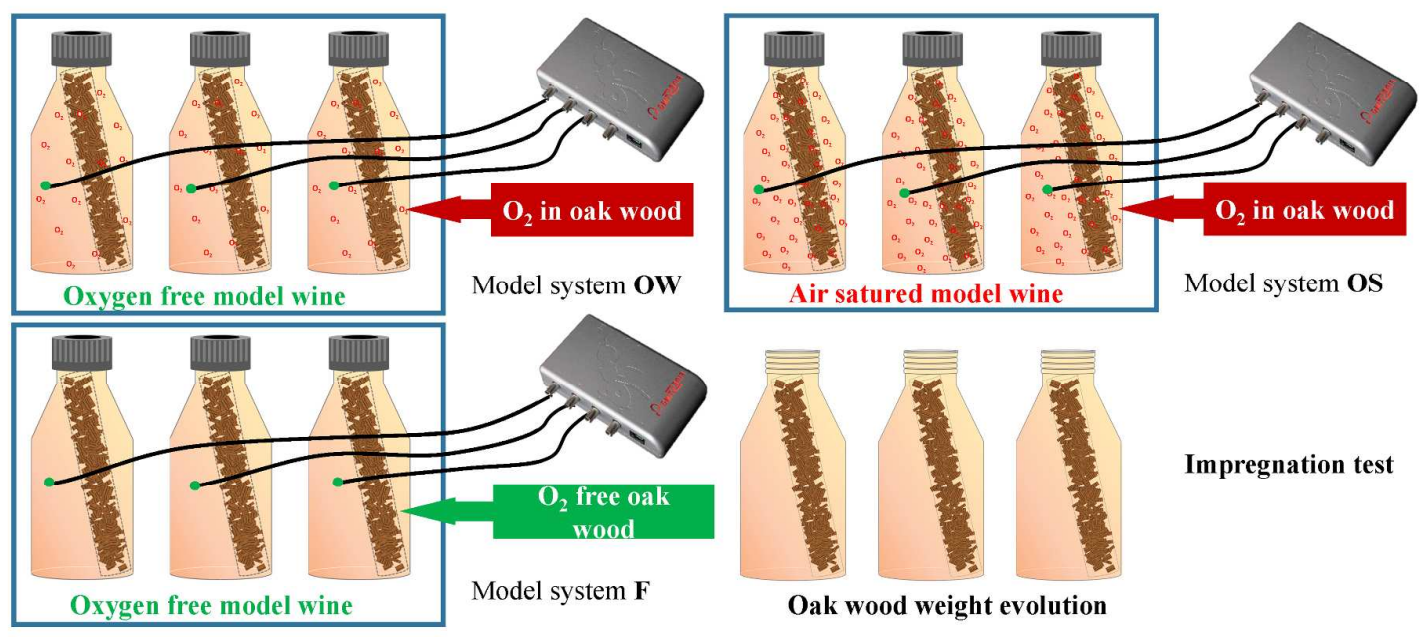




\section{Figure 2.}
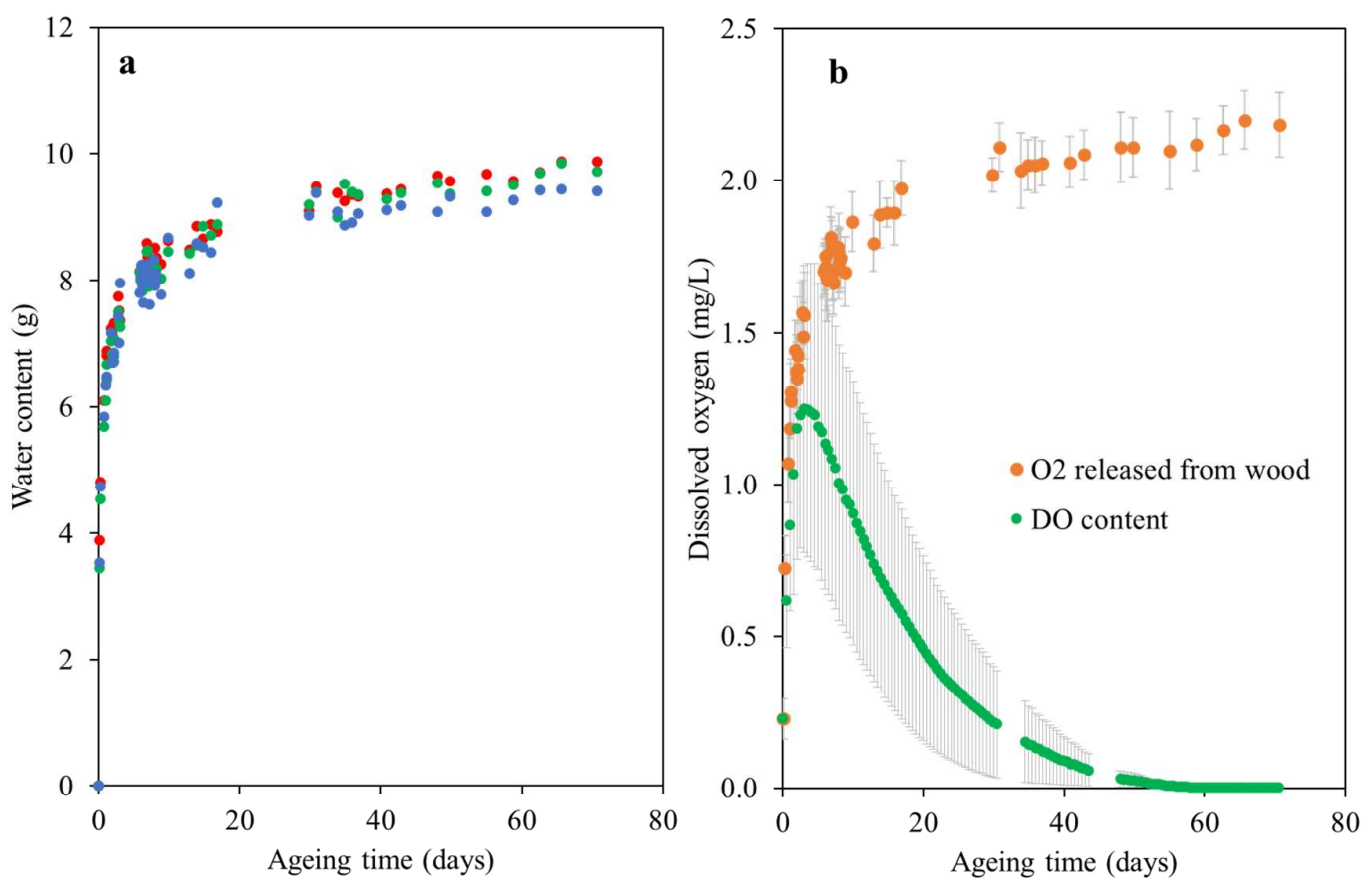


\section{Figure 3.}
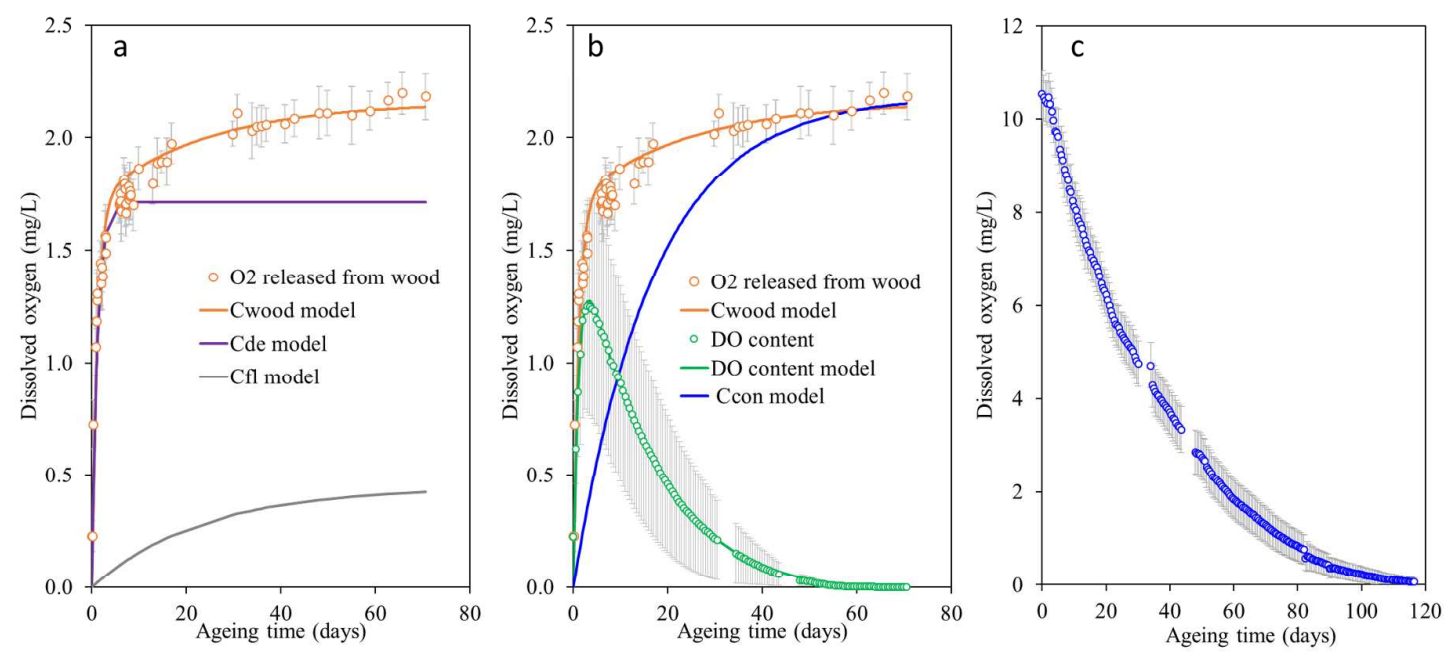


\section{Figure 4.}

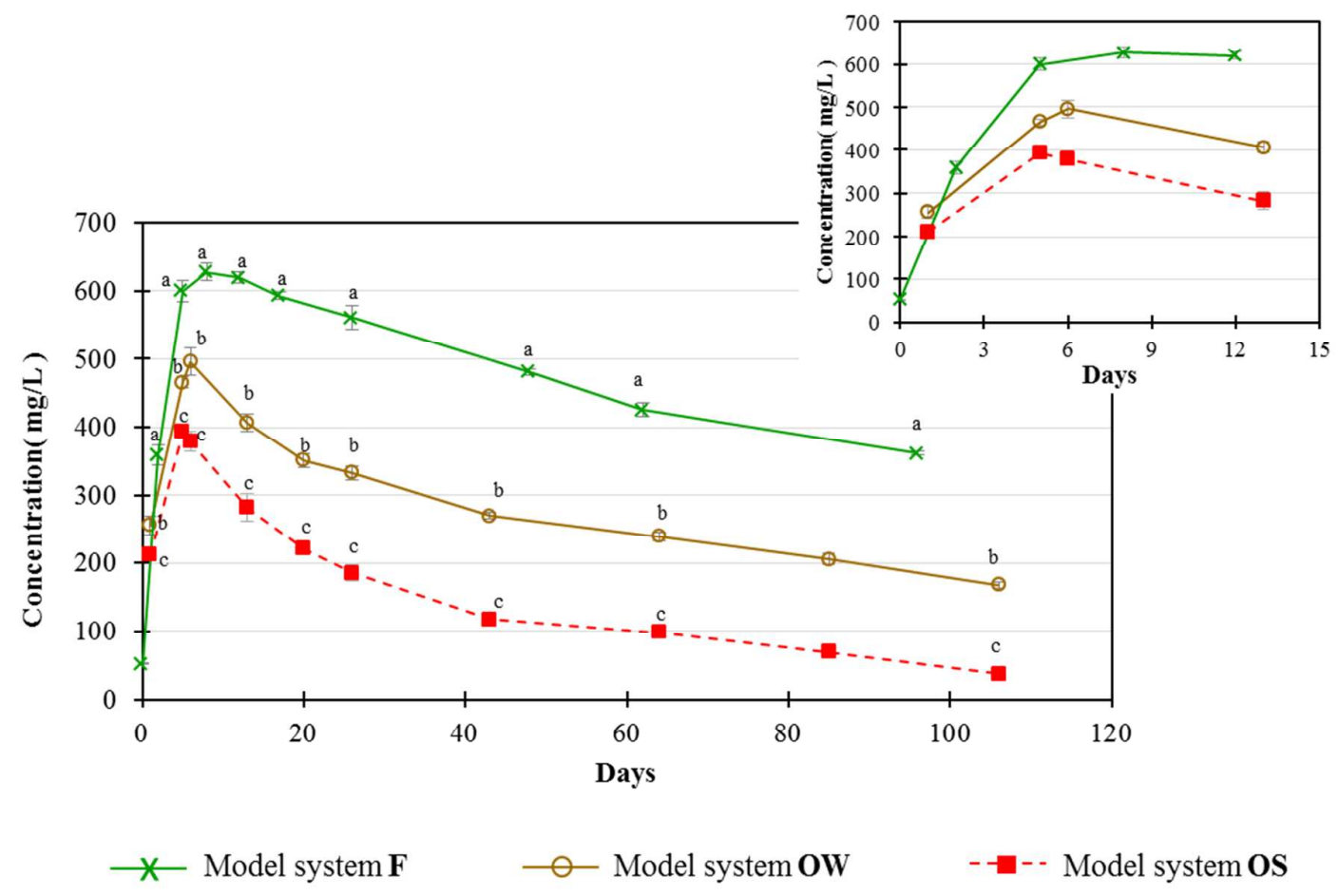




\section{Figure 5.}
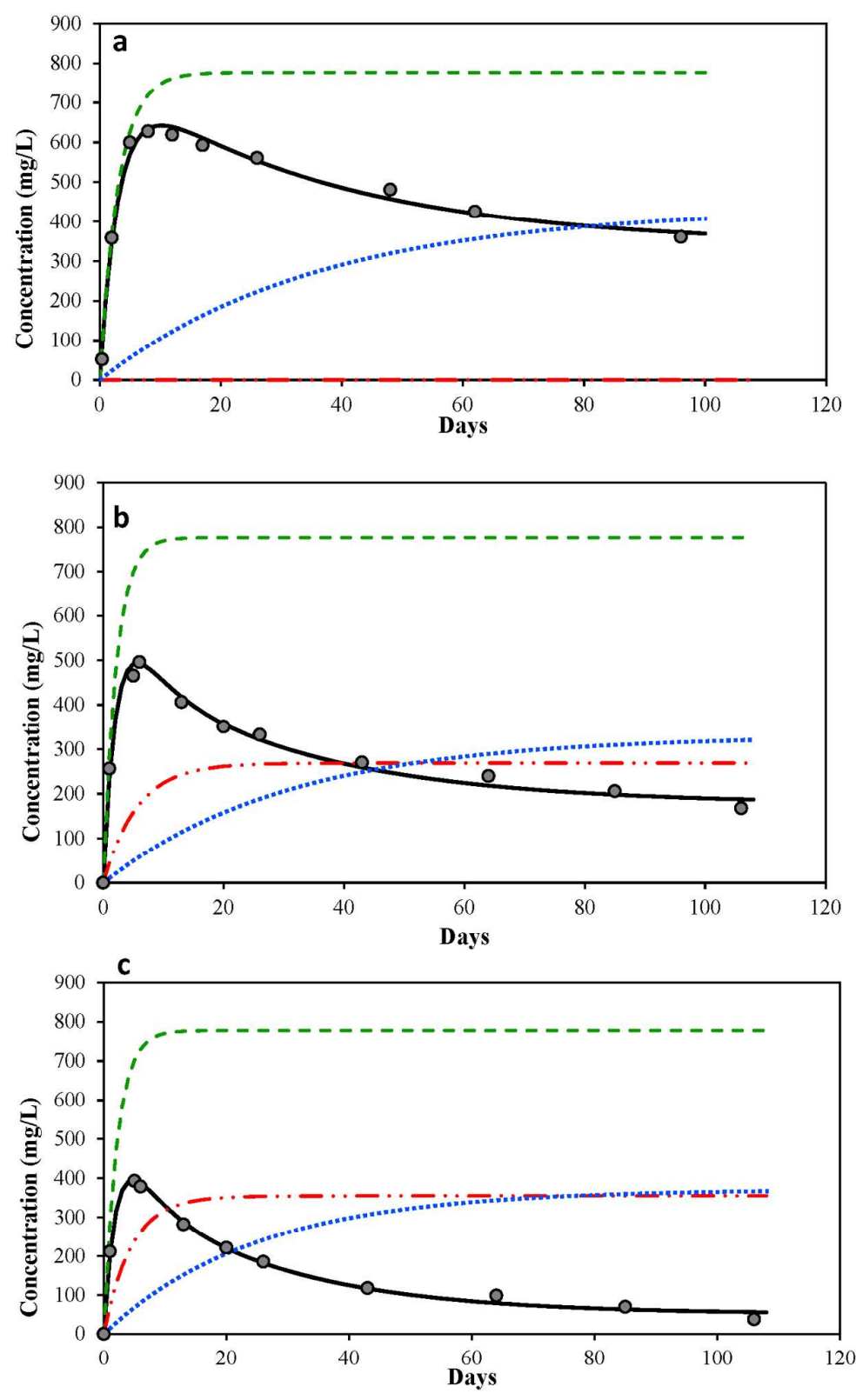

○ Real —Model - - Ext - - . Ox 


\section{Figure 6.}
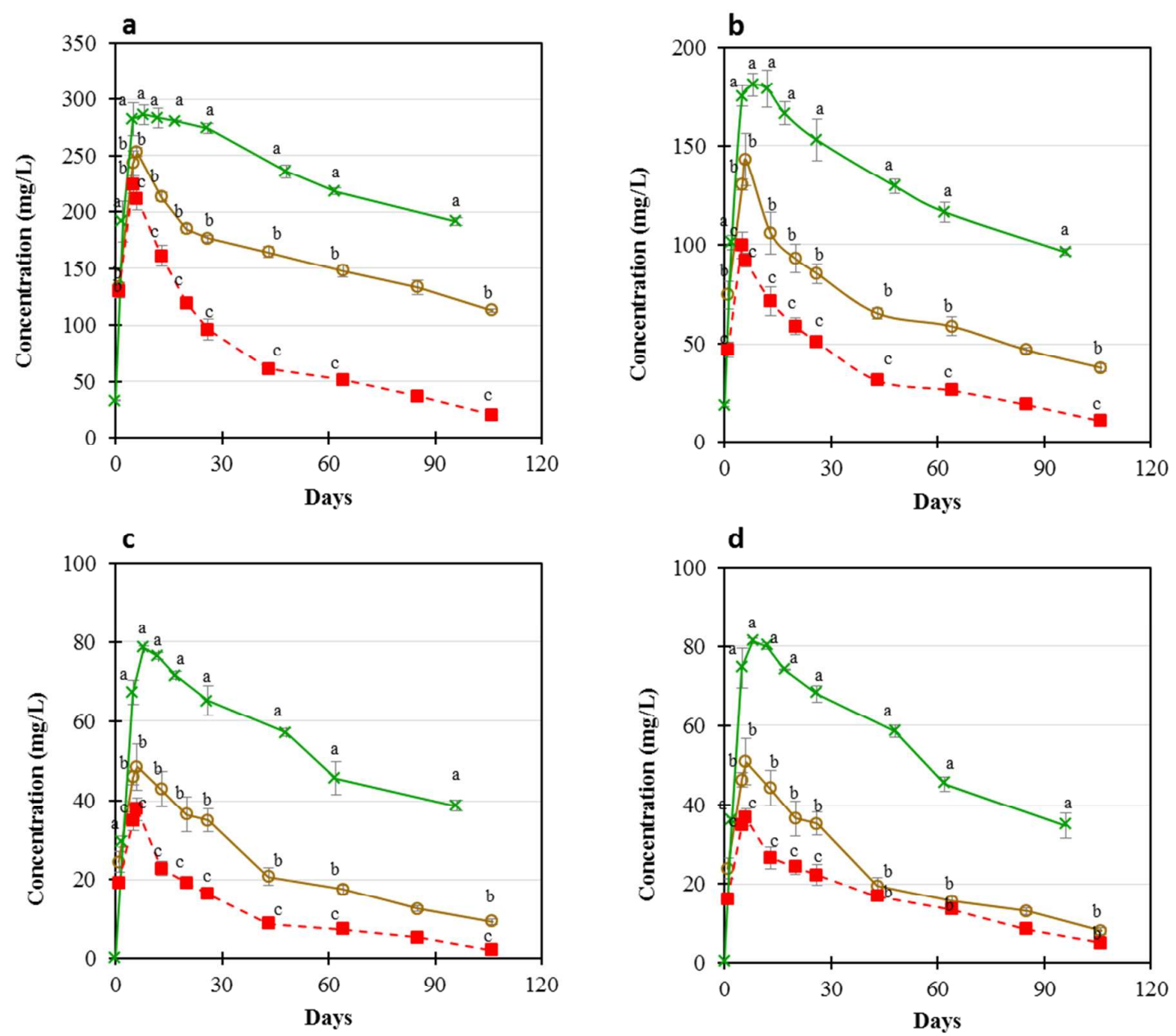

$\longrightarrow$ Model system $\mathbf{F}$ 
For Table of Contents Only: TOC

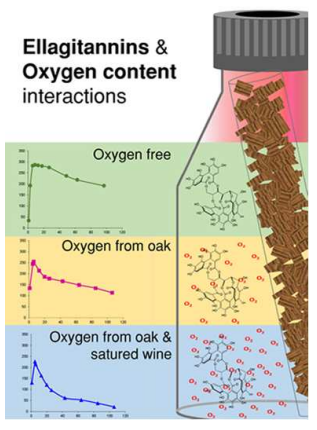

